\title{
Indicator cell lines for the detection of hidden mycoplasma contamination, using an adenosine phosphorylase screening test
}

\author{
Gerrit T. Spierenburg ${ }^{1}$, Anna A. Polak-Vogelzang ${ }^{2}$ and Bert J.E.G. Bast ${ }^{1}$ \\ ${ }^{\prime}$ Department of Clinical Immunology, University Hospital, Utrecht, The Netherlands, \\ and ${ }^{2}$ National Institute of Public Health and Environmental Hygiene, Bilthoven, The Netherlands \\ (Received 19 January 1988, revised received 25 April 1988, accepted 23 May 1988)
}

Mycoplasmas are a major cause of cell culture contamination and are especially troublesome during HAT selection. The enzyme adenosine phosphorylase (adoP) is present in all common mycoplasma species but is considered to have a low activity in mammalian cells. However, using an adoP screening test, we have observed that some cell cultures do possess an intrinsic adoP activity leading to false positive results. Moreover, as a false negative result, we encountered a variant of Mycoplasma orale (identified after cultivation on agar and immunostaining) which was not detectable with the adoP screening in cell culture supernatants and only at low levels in cell lysates. To increase the low signal/noise adoP ratio found there, we used an indicator cell line with low intrinsic activity. Indicator cells were inoculated with the test supernatant and the adoP activity of these infected cells were measured after lysis. The procedure diminished the effect of biological variation in intrinsic enzyme activity between the several cell lines tested. Furthermore, in another mycoplasma infected cell line (with $M$. fermentans), this infection was only reliably detected using these indicator cells. With this procedure we obtained rapid results which were concordant with those obtained using the time consuming cultivation on agar.

Key words: Adenosine phosphorylase activity; Hidden mycoplasma infection; Indicator cell line procedure

\section{Introduction}

Mollicutes (mycoplasmas and acholeplasmas) are small, free-living unwalled prokaryotes that often contaminate cell cultures. From the 20 different mollicute species isolated from cell cultures, originating from bovine, human and porcine

Correspondence to: G.T. Spierenburg, Department of Clinical Immunology, University Hospital, Catharynesingel 101, 3511 GV Utrecht, The Netherlands.

Abbreviations: adoP, adenosine phosphorylase; CFU, colony forming units; DAPI, 4,6-diamidino-2-phenylindole-2$\mathrm{HCl}$; HAT, hypoxanthine, aminopterine, thymidine; $\mathrm{M}$, mycoplasma. sources, four of them, Mycoplasma orale, $M$. arginini, $M$. hyorhinis and Acholeplasma laidlawii, account for approx. $80 \%$ of the contaminations (McGarrity et al., 1986).

Contamination may result in cell death or reduction of growth rate. In immunological research the effects of mycoplasma infection can be dramatic: for instance, the induction of proliferation and maturation of $\mathrm{B}$ and $\mathrm{T}$ cells by mycoplasmas or their products (Mizushima et al., 1985; Proust et al., 1985) and disturbed hybridoma HAT selection (Shin and Van Diggelen, 1978) have been described. Therefore, it is clear that not only cell cultures but also their products have to be tested for mycoplasma contamination. Several techniques have been described, most of which are 
neither simple nor rapid, and, in some cases, may require specialized equipment or skills. Also false negative or false positive results have been obtained (McGarrity et al., 1986; Polak-Vogelzang et al., 1987; this report).

Purine nucleoside phosphorylase catalyzes the phosphorolysis of the nucleosides of both hypoxanthine and guanine. In addition, in mollicutes but according to Zimmerman et al. (1971) not in mammalian cells, adenosine is a substrate for this enzyme. In the case of the microorganism adenine and ribose-1-phosphate are produced. The enzyme responsible, which also catalyses the opposite reaction, is called adenosine phosphorylase (adoP) and has been detected in a variety of mollicutes. Therefore, the measurement of adoP activity permits the specific detection of mycoplasma contamination in cell cultures (Hatanaka et al., 1975; McGarrity et al., 1986). An indicator cell line procedure improves the sensitivity and specificity of the test (McGarrity and Barile, 1983).

In this paper we confirm the advantages of the simple and rapid biochemical test that uses adoP activity as a marker for mycoplasma contamination. However, depending on the assay system, both false negative and false positive results can occur. False negatives, when caused by low intensities of contamination, can be eliminated by measuring adoP activity in cell extracts. However, we found that due to a high intrinsic enzyme activity in some cell cultures, this can also introduce false positive results. A combination of the advantages of both the biochemical test for measuring adoP activity in cell lysates and the use of an indicator cell line seems to be the basis of an optimal mycoplasma screening test. The advantages of this approach became clear when we encountered a hidden $M$. orale with an extremely low intensity of contamination.

\section{Materials and methods}

\section{Cell cultures}

All cell cultures were cultivated in RPMI 1640 $\mathrm{N}$-2-hydroxyethyl-piperazine- $\mathrm{N}$-2-ethanesulphonic acid (Hepes)-buffered medium (Gibco, The Netherlands) supplemented with $10 \%$ foetal calf serum (Gibco), $100 \mathrm{U} / \mathrm{ml}$ penicillin and 100 $\mu \mathrm{g} / \mathrm{ml}$ streptomycin. The $3 \mathrm{~T} 3$ mouse embryo fibroblast culture was obtained from Gibco.

\section{Mycoplasma detection procedures}

Cultivation on agar and the DAPI-DNA fluorescence test were performed as described by Polak-Vogelzang et al. (1987). The number of colony forming units (CFU)/ml was determined by cultivation on agar of serially diluted samples. When incubation on agar resulted in mycoplasma colonies, they were identified with an indirect immunofluorescence test using polyclonal antibodies against various mycoplasma species (Rosendal and Black, 1972).

Adenosine phosphorylase activity (adoP) was determined according to Hatanaka et al. (1975) in supernatants and cell extracts, with minor modifications. $100 \mu 1$ supernatant was incubated with 10 $\mu \mathrm{l}\left[{ }^{3} \mathrm{H}\right]$ adenosine (Amersham TRK 423;5 $\mu \mathrm{M}$ ) + $10 \mu 1$ erythro-9-(2-hydroxy-3-nonyl)-adenine (EHNA, an inhibitor of adenosine deaminase; a generous gift from Burroughs-Wellcome; $120 \mu \mathrm{M}$ ). $20 \mu 1$ cell extract $\left(2 \times 10^{5}\right.$ lyophilised cells $)$ was incubated with $5 \mu 1\left[{ }^{14} \mathrm{C}\right.$ ]adenine (Amersham CFA $348,0.1 \mu \mathrm{M})+5 \mu 1$ adenine $(0.66 \mu \mathrm{M})+5 \mu 1$ ribose-1-phosphate $(10 \mathrm{mM})+5 \mu 1$ buffer $(1 \mathrm{M}$ Tris $+0.1 \mathrm{M}$ EDTA pH 7.4). After 1.5 and $3 \mathrm{~h}$ of incubation $\left(37^{\circ} \mathrm{C}\right)$ adenosine and adenine were separated: $1.5 \mu 1$ of the reaction mixture was spotted on polyethyleneimine (PEI) cellulose (Macherey-Nagel, Dueren, F.R.G.; art. no. 801053). After eluting with $0.15 \mathrm{M} \mathrm{NaCl}$ the spots were identified using reference compounds under UV light $(254 \mathrm{~nm})$, cut out and counted in a liquid scintillation counter.

\section{Indicator cell line}

Test supernatant containing several hundred cells was inoculated into a mycoplasma-free indicator cell line with low intrinsic adoP activity. After 3 days of culturing adoP activity was determined in the supernatant or cell extract as described above.

\section{Results}

Using the adoP-supernatant assay over 2 years we tested over 100 cell lines for evidence of con- 
tamination with mycoplasma. We detected contamination in 25 out of 115 cultures, derived from 11 out of 26 laboratories. From eight laboratories found to have infection we received two or more different cultures: in six of these laboratories at least two cell lines were infected.

Cell lines in which no adoP activity in the supernatant was detected were declared admissible for use in our laboratory. One cell line, however, caused problems in HAT selection after cell fusion. Using the agar cultivation method, it appeared that this cell line was indeed contaminated with mycoplasma. This particular strain proved to be $M$. orale as determined by immunofluorescence staining. Furthermore, three other cell lines (from the same laboratory) were cross-contaminated. Further investigations of the $M$. orale-contaminated cell line anti-PO 3 showed that (low) adoP activity was demonstrable in the cell extract, but that the mycoplasma was missed by DAPIDNA staining (Table I). These results indicated a low intensity of $M$. orale contamination, confirmed by the low numbers of $\mathrm{CFU} / \mathrm{ml}$ found (Table I).

\section{TABLE I}

DETECTION OF HIDDEN $M$. ORALE CONTAMINATION

\begin{tabular}{|c|c|c|}
\hline & Cell line: & anti-PO 3 \\
\hline & ycoplasma infection: & M. orale \\
\hline Mycoplasma detection ${ }^{\mathbf{a}}$ & $\begin{array}{l}\text { DAPI } \\
\text { agar } \\
\text { adoP sup. (anti-PO3) } \\
\text { adoP sup. (Ag8.653) } \\
\text { adoP extr. (anti-PO3) } \\
\text { adoP extr. (Ag8.653) }\end{array}$ & $\begin{array}{l}- \\
+ \\
-(9.5 \%) \\
-(11.0 \%) \\
+(22.2 \%) \\
+(25.9 \%)\end{array}$ \\
\hline Number of $\mathrm{CFU} / \mathrm{ml}$ & $\begin{array}{l}\text { Supernatant }+ \text { cells } \\
\text { Supernatant }\end{array}$ & $\begin{array}{l}10-100 \\
<10\end{array}$ \\
\hline
\end{tabular}

a Detection procedures: DAPI, DAPI-DNA fluorescence staining; agar, cultivation on agar; adoP sup., adoP activity in supernatant of anti-PO 3 or of the indicator cell line Ag8.653 after inoculation with test supernatant; adoP extr., adoP activity in cell extract of anti-PO 3 or of the indicator cell line $\mathrm{Ag} 8.653$ after inoculation with test supernatant. -, no contamination; +, contamination. In brackets the adoP activity is expressed as $\%$ product formed after $3 \mathrm{~h}$ of incubation.

b Samples contained approx. $5 \times 10^{5}$ cells $/ \mathrm{ml}$ and were frozen at $-70^{\circ} \mathrm{C}$ and thawed once. Usually, contaminated cell cultures contained approximately $1-10 \times 10^{6} \mathrm{CFU} / \mathrm{ml}$.

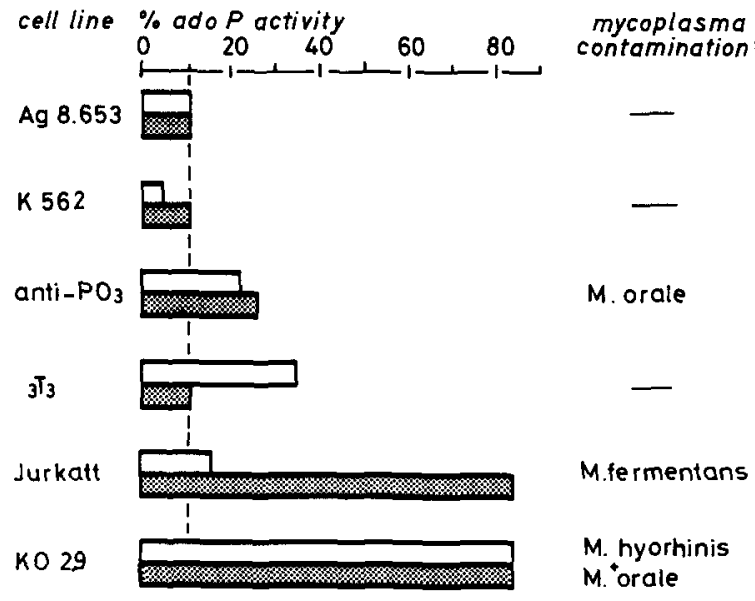

Fig. 1. Mycoplasma contamination using an indicator cell line. adoP activity was measured in the extract of the individual cell lines (open bar) or in the extract of the indicator cell line Ag8.653 after inoculation with test supernatant (closed bar). The dotted line represents the intrinsic activity of the indicator cell line Ag8.653. The activity is expressed as \% product formed after $3 \mathrm{~h}$ of incubation. Note the false negative result (Jurkatt) and false positive result (3T3) obtained if only adoP activity in individual cell lines was determined.

* Detection methods used; DAPI-DNA fluorescence test and cultivation on agar followed by identification by immunofluorescence.

\section{Indicator cell lines}

Measuring adoP activity in some cell extracts of various mycoplasma-free cell cultures revealed a variation in intrinsic enzyme activity. Inoculation of a mycoplasma-free indicator cell line with test supernatant reduced this variation. This observation is of great importance when dealing with cell lines that have high intrinsic adoP activity such as 3T3 mouse embryo fibroblasts (Fig. 1). Furthermore, in extracts of the $M$. fermentanscontaminated Jurkatt cell culture a low adoP activity was determined, whereas after inoculation of the supernatant of this mycoplasma contaminated culture to the indicator cells Ag8.653, a significantly higher adoP activity was measured in cell lysates (Fig. 1).

\section{Discussion}

Mycoplasma is a major cause of contamination and loss of cell cultures and can have unpredictable effects. The infection is often introduced by 
cross-contamination from other cell lines already suffering from mycoplasma contamination. Because most laboratories do not have the facilities for culturing mycoplasma-suspected cell lines under quarantine conditions (separated laminar air flow hood and incubator) a rapid, sensitive detection procedure is required. Biochemical and DNA staining procedures do offer these advantages, although the latter sometimes yields false negatives (Table I, and Polak-Vogelzang et al., 1987). A frequently used biochemical detection procedure is based on adenosine phosphorylase activity (Hatanaka et al., 1975).

The enzyme adenosine phosphorylase (adoP) is present in almost all mycoplasma species and has low intrinsic activity in mammalian cells (Zimmerman et al., 1971). However, we found some activity in a $3 \mathrm{~T} 3$ mouse embryo fibroblast culture (Fig. 1). The intrinsic activity measured in the mycoplasma-free (as determined via cultivation on agar and DAPI-DNA staining) $3 T 3$ culture was even higher than the adoP activity found in the mycoplasma-contaminated (see above) anti-PO 3 hybridoma and Jurkatt cell culture. The use of an indicator cell line with low intrinsic activity evidently resolved this problem (Fig. 1).

Usually, in a contaminated cell culture the number of mycoplasma particles exceeds that of the cells by a factor of 100 (McGarrity et al., 1984). In this paper, however, we describe a $M$. orale contamination that contained about one particle per $10^{3}$ cells. Such a low intensity of contamination requires an extremely sensitive test system. Apparently neither the DAPI-DNA fluorescence test (using cells plus supernatant) nor the adoP activity screening using cell supernatant meet this requirement (Table I). In spite of the low number of particles found, it is possible to inoculate an (indicator) cell line with $M$. oralecontaminated supernatant, as was shown by the indicator cell line procedure (Fig. 1).

Measuring adoP activity in cell extracts, mycoplasma contamination is readily detectable (Hatanaka et al., 1975; and KO 2.9 in Fig. 1). This detection is dependent on both the intensity of mycoplasma contamination and on the adherence of the mycoplasma particles to the cell surface. The two hidden mycoplasma contaminations, as described in this paper, illustrate those different problems in detection. In supernatants of the $M$. fermentans-contaminated Jurkatt cell culture and the Ag8.653 indicator cell cultures infected with the supernatant of the infected Jurkatt culture, a high adoP activity was measured (data not shown): apparently indicating contamination. In extracts of the Jurkatt cell culture, however, a false negative result was obtained, possibly caused by low adherence of the mycoplasma particles to the Jurkatt cells, whereas the $M$. fermentans infection of the indicator cell line Ag8.653 resulted in high adoP activity in these indicator cells (Fig. 1). Another hidden mycoplasma, $M$. orale in the contaminated anti-PO3 hybridoma (Fig. 1), was hardly detectable, even in indicator cells. In this case the intensity of contamination appeared to be low (see CFU, Table I).

From these results, it can be concluded that the combination of both adoP activity screening in cell extracts and the use of an indicator cell line provides a rapid, sensitive and reliable screening test for mycoplasma contamination.

\section{Acknowledgements}

We are most grateful to J. Brugman and R. Reijgers for their technical assistance in the DAPI-DNA fluorescence test and the procedure of cultivation on agar.

\section{References}

Hatanaka, M., Del Giudice, R. and Long, C. (1975) Adenine formation form adenosine by mycoplasmas: adenosine phosphorylase activity. Proc. Natl. Acad. Sci. U.S.A. 72, 1401.

McGarrity, G.J. and Barile, M.F. (1983) Use of indicator cell lines for recovery and identification of cell culture mycoplasmas. In: J.G. Tully and S. Razin (Eds.), Methods in mycoplasmology, Vol. 2. Academic Press, New York, p. 167.

McGarrity, G.J., Vanaman, V. and Sarama, J. (1984) Cytogenetic effects of mycoplasmal infection of cell cultures: a review. In Vitro 20, 1.

McGarrity, G.J., Kotani, H. and Carson, D. (1986) Comparative studies to determine the efficiency of 6-methylpurine deoxyriboside to detect cell culture mycoplasmas. In Vitro Cell. Dev. Biol. 22, 301.

Mizushima, Y., Quintans, J. and Cohen, E.P. (1985) Stimula- 
tion of lymphoid cell proliferation by Mycoplasma orale, a common cell culture contaminant. Infect. Immun. 50, 636.

Polak-Vogelzang, A.A., Brugman, J. and Reijgers, R. (1987) Comparison of two methods for detection of Mollicutes (Mycoplasmatales and Acholeplasmatales) in cell cultures in The Netherlands. Antonie van Leeuwenhoek J. Microbiol. $53,107$.

Proust, J.J., Buchholz, M.A. and Nordin, A.A. (1985) A 'lymphokine-like' soluble product that induces proliferation and maturation of $B$ cells appears in the serum-free supernatant of a $\mathrm{T}$ cell hybridoma as a consequence of mycoplasmal contamination. J. Immunol. 134, 390.
Rosendal, S. and Black, F.T. (1972) Direct and indirect immunofluorescence of unfixed and fixed mycoplasma colonies. Acta Pathol. Microbiol. Scand. 80B, 615.

Shin, S. and Van Diggelen, O.P. (1978) Phenotypic alterations in mammalian cell lines after mycoplasma infection. In: G.J. McGarrity, D.G. Murphy and W.W. Nichols (Eds.), Mycoplasma infection of cell cultures, Vol. 3. Plenum Press, New York, p. 191.

Zimmerman, T.P., Gersten, N.B., Ross, A.F. and Miech, R.P. (1971) Adenine as substrate for purine nucleoside phosphorylase. Can. J. Biochem. 49, 1050. 